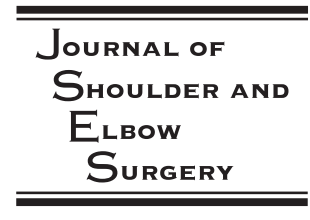

www.elsevier.com/locate/ymse

\title{
Arthroscopic versus open release of internal rotation contracture in the obstetrical brachial plexus paralysis (OBPP) sequela
}

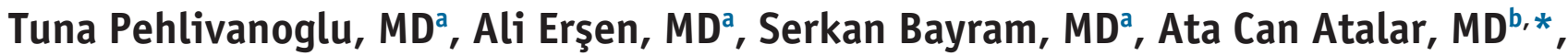 \\ Mehmet Demirhan, MDc
}

\author{
${ }^{a}$ Department of Orthopaedic Surgery and Traumatology, Istanbul Faculty of Medicine, Istanbul University, Istanbul, Turkey \\ ${ }^{b}$ Department of Orthopaedic Surgery and Traumatology, Faculty of Medicine, Aclbadem University, Istanbul, Turkey \\ ${ }^{c}$ Department of Orthopaedic Surgery and Traumatology, School of Medicine, Koç University, Istanbul, Turkey
}

\begin{abstract}
Background: Latissimus dorsi (LD) and teres major (TM) tendon transfers are effective surgical procedures to improve shoulder abduction and external rotation for children with obstetrical brachial plexus palsy (OBPP). Open pectoralis major (PM) tendon Z-plasty and arthroscopic subscapularis (SS) release are 2 options for the release of internal rotation contractures to enhance muscle transfers. This study compared the functional results of LD and TM tendon transfers with open PM tendon Z-plasty or arthroscopic SS release.

Methods: The study included 24 patients who underwent LD and TM tendon transfers for OBPP (9 arthroscopic SS release, 15 open PM tendon Z-plasty) with a mean follow-up of 41.33 months (range, 3660 months) and 47.2 months (range, 36-60 months), respectively. Functional evaluation was made according to range of motion and Mallet scoring system.

Results: Shoulder abduction-external rotation degrees and scores in all sections of the Mallet scoring system significantly increased in both groups $(P<.001)$. Postoperatively, the arthroscopic SS release group had significantly better abduction degrees $(P=.003)$, total Mallet scores $(P<.001)$, and superior abduction $(P=.043)$, active external rotation $(P=.043)$, hand-to-head $(P=.043)$, and hand-to-mouth $(P<.001)$ scores for the Mallet scoring system.

Discussion: Transfer of LD together with TM tendons combined with one of the internal rotation contracture release procedures yielded good clinical and functional results in patients younger than age 7 , regardless of the type of release method. However, arthroscopic SS release, although requiring an experienced surgeon, revealed better clinical and functional outcomes and is considered to be a less invasive and superior method.
\end{abstract}

\footnotetext{
This study was presented as a free-paper at the XXVI Congress of the European Society for Shoulder and Elbow Surgery, Milan, Italy, September 16-19, 2015.

The Istanbul University, Istanbul Faculty of Medicine, Department of Orthopedic Surgery and Traumatology Institutional Review Board approved this study (registry number 1210037).
}

\footnotetext{
*Reprint requests: Ata Can Atalar, MD, Acıbadem University, Faculty of Medicine, Department of Orthopaedic Surgery and Traumatology, Büyükdere Cd. No. 40 Maslak, Sarıyer 34457, Istanbul, Turkey.

E-mail address: atacan.atalar@acibadem.edu.tr (A.C. Atalar).
}

1058-2746/\$ - see front matter ( 2018 Published by Elsevier Inc. on behalf of Journal of Shoulder and Elbow Surgery Board of Trustees. 
Level of evidence: Level III; Retrospective Cohort Comparison; Treatment Study

(C) 2018 Published by Elsevier Inc. on behalf of Journal of Shoulder and Elbow Surgery Board of Trustees.

Keywords: Obstetrical brachial plexus palsy; arthroscopic SS release; open pectoralis major tendon Z-plasty; latissimus dorsi and pectoralis major tendon transfers; internal rotation contracture; release procedures; Mallet score

Obstetric brachial plexus palsy (OBPP), as one of the most dramatic disabilities after birth, ${ }^{34,39}$ has a reported incidence of $0.1 \%$ to $0.4 \% .^{10,40,43}$ Although spontaneous recovery was noted in $50 \%$ to $92 \%$ 3,24,38,40 of cases, a high risk of longterm morbidity was underlined in patients without recovery. ${ }^{10,17,21,26,27,35,38,42}$

To define the problem and provide solutions, clinical and radiologic classification systems have been identified. 2,4,9,45,46 The involvement of C5-C6 $\pm \mathrm{C} 7$ roots, also described as Erb's palsy, mostly results in progressive problems of the glenohumeral joint. ${ }^{3,5,9,21,27,44}$

For patients with OBPP, regardless of the treatment in infancy, contractures of internal rotation, together with the weakening of external rotators that leads to progressive bony deformities due to the dynamic phenomenon of muscle imbalance, are likely to develop in the following years. ${ }^{4,13,19,21,32,35,38,42,46}$ In untreated cases, this problem results in the shortening of the dominant muscles of the shoulder, including the pectoralis major (PM), subscapularis (SS), and latissimus dorsi (LD). This may lead to secondary changes of the bone and joint, including glenoid hypoplasia, retroversion, and flattening of the humeral head, which result in posterior subluxation. ${ }^{12,13,21,22,25,27,36,38,44}$ Among the most commonly seen results of OBPP are serious limitations in abduction and external rotation due to the paresis of the external rotators and abductors and the added unopposed cocontraction of the internal rotators together with adductors leading to internal rotation contracture. . $^{2,3,8,10,11,13,15,32,37,38,41,45}$

The mainstay of treatment is to reconstruct the muscular balance and restore shoulder function concomitantly. ${ }^{4,27,39}$ Various surgical options have been defined, including tendon transfers, muscle-tendon releases, and bony procedures, such as humeral derotational osteotomies. ${ }^{4,6,8,9,15,27,33,38,40,44}$ The Hoffer et $\mathrm{al}^{20}$ modification of L'Episcopo's technique of transferring the teres major (TM) and LD to the rotator cuff as high as possible improved the stabilizing effect of the rotator cuff by yielding a higher glenohumeral abduction when there was a functioning deltoid. ${ }^{2,9,15,28,34,38,45}$ Fairbank $^{16}$ introduced the SS tendon release with anterior capsulotomy in 1913 with high rates of complications. Sever ${ }^{33}$ modified this technique by removing the capsulotomy and only performing the SS tendon release while reporting loss of active and passive internal rotation in his series, which is also supported by Pichon et al in their study. ${ }^{31}$ Carlioz and Brahimi ${ }^{7}$ described the proximal SS release in 5 patients before the apperance of glenohumeral deformity and reported good results. Some authors applied open or arthroscopic SS release in addition to these tendon transfers. ${ }^{3,10,14,38-40}$ A full arthroscopic SS release was performed by Pearl, ${ }^{29}$ whereas Pedowitz et $\mathrm{al}^{30}$ performed a partial release, and Kany et $\mathrm{al}^{23}$ prefer the release of superior glenohumeral and coracohumeral ligament only, without releasing the SS tendon. PM tendon lengthening was also preferred by some authors. ${ }^{3,10,15,27,38}$

This study compared the effectiveness of arthroscopic SS release vs. open PM tendon Z-plasty added to the transfer of LD and TM tendons to the rotator cuff in clinical and functional results in patients with OBPP aged younger than 7 years. A comparative, retrospective study of 24 patients was conducted.

\section{Materials and methods}

Between 2009 and 2013, 54 patients underwent surgery in our institution because of shoulder problems resulting from OBPP, and 24 patients of this group were included in the study. The inclusion criteria were age 7 years or younger, a diagnosis of Erb palsy, and having undergone LD plus TM tendon transfer, together with internal rotation contracture release procedures (arthroscopic SS release or open PM tendon Z-plasty). The study excluded 30 patients older than 7 years, with lower trunk palsies, who had been treated with derotational osteotomies and transfers without releases. All patients and their parents provided informed consents so that their operative, intraoperative, and postoperative data, including the photos and videos, could be used for publication by hiding their identity.

As a result of preoperative radiographic evaluation, no posterior dislocation was detected in any patient enrolled in the study.

The study population comprised 24 patients ( 15 boys and 9 girls), with a mean age of 5.87 years and a mean follow-up time of 44.26 months (range, 36-60 months), who underwent LD and TM tendon transfers. Arthroscopic SS release was performed in 9 patients (group 1), and 15 patients underwent open PM tendon Z-plasty (group 2). Whether the type of release performed was open or arthroscopic was mainly based on the preference of 2 senior surgeons (A.C.A., M.D.).

Patients in group 1 were a mean age of 3.88 years and had a mean follow-up time of 41.33 months (range, 36-60 months). Patients in group 2 were a mean age of 3.93 years and had a mean follow-up time of 47.2 months (range, 36-60 months; Table I).

Arthroscopic SS release was conducted with the patient in lateral decubitus, with a $2.7-\mathrm{mm}$ scope and a radiofrequency probe. The long head of biceps tendon was identified immediately after entering into the glenohumeral joint, followed by the release of the anterior capsule, glenohumeral ligaments, and intra-articular portion of SS tendon while a full SS tenotomy was performed as described by Pearl et $\mathrm{al}^{29}$ (Fig 1).

Open PM tendon Z-plasty was also conducted with the patient in lateral decubitus through a deltopectoral incision. After the exposure of the PM, tenotomy of the clavicular head was undertaken at the insertion point to the humerus. The sternal head was tenotomized at the musculotendinous junction, followed by a Z-plasty to the free tendon ends as described by Ozben et al. ${ }^{27}$ 
Table I Demographic values of both patient groups

\begin{tabular}{lccc}
\hline Variables & Arthroscopic SS release group & & Open PM Z-plasty group \\
\cline { 2 - 3 } & Mean \pm SD (min-max) & Mean \pm SD (min-max) & $3.93 \pm 0.88(2-5)$ \\
\hline Age, yr & $3.88 \pm 1.05(2-5)$ & $47.2 \pm 11.53(36-60)$ & .975 \\
Follow-up, mo & $41.33 \pm 8.71(36-60)$ & $10.73 \pm 1.03(10-14)$ & .290 \\
Preoperative Mallet score & $10.67 \pm 0.7(10-12)$ & & .907 \\
\hline SS, subscapularis; PM, pectoralis major; SD, standard deviation. & \\
* $P$ values were calculated by Mann-Whitney U test.
\end{tabular}
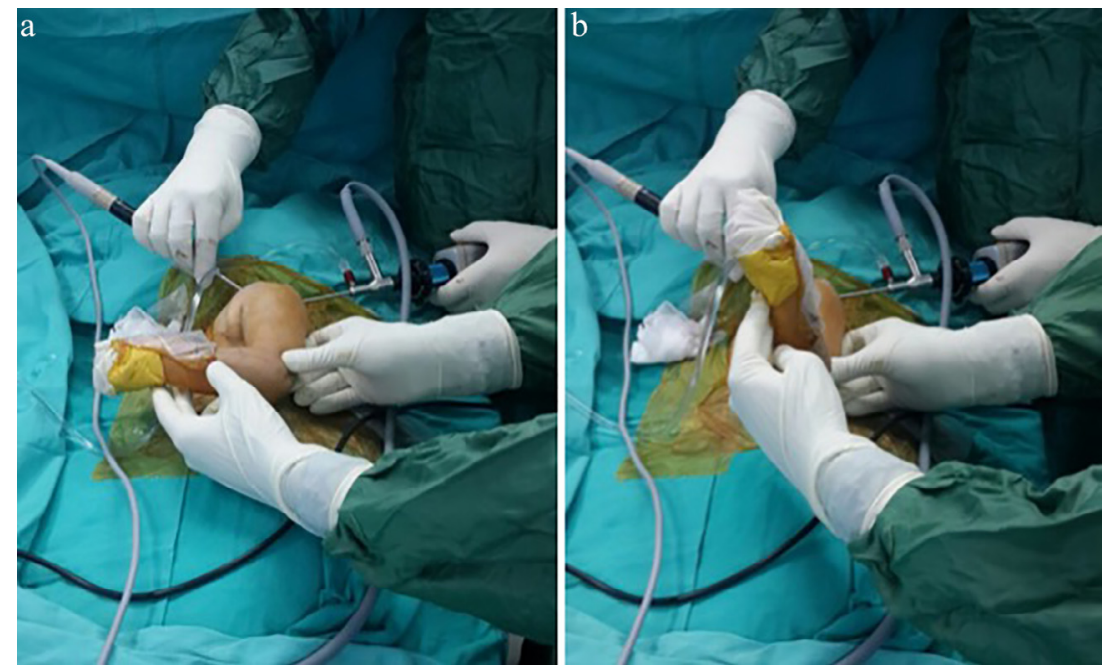

Figure 1 (a) Arthroscopic setup for subscapularis release in a 2-year-old patient with obstetrical brachial plexus paralysis with marked internal rotation contracture. (b) Achievement of passive full external rotation after the completion of arthroscopic subscapularis release for the same patient.

In both groups, LD together with TM tendon transfers to supraspinatus and infraspinatus by using posterior axillary incisions were added to the surgical procedures.

All of the patients were maintained in a preoperatively constructed brace $\left(60^{\circ}\right.$ abduction and maximum external rotation of the shoulder) for 6 weeks. After the brace was removed at week 6, all patients began a physical therapy program with gradually increasing active range of motion.

Patients were evaluated functionally using the Mallet scoring system preoperatively and postoperatively during the latest followup visit. The Mallet scoring system consists of the 5 categories of abduction, extension, hand to head, hand to mouth, and hand to back, where a maximum score of 20 could be obtained. Patients were monitored radiographically using $\mathrm{x}$-ray imaging and magnetic resonance imaging, if available.

The statistical analysis was performed with SPSS 22.0 software (IBM, Armonk, NY, USA). For quantitative variables between the 2 groups, the Student $t$ test was used. Data are expressed as mean \pm standard deviation. The $\chi^{2}$ test and Fisher exact test were used for the analysis of categoric variables, where appropriate. A $P$ value of $<.05$ was considered as statistically significant.

\section{Results}

Group 1 and 2 were comparable in age, number, and preoperative Mallet score status $(P>.05)$. Mallet scores of all groups were improved significantly at the latest followup visit.

Group 1 (patients with only arthroscopic SS release in addition to LD and TM tendon transfers) had a statistically highly significant increase in scores of abduction (preoperative: 2, postoperative: $3.89 ; P<.001$ ), external rotation (preoperative: 2.33 , postoperative $3.89 ; P<.001$ ), hand to head (preoperative: 2.11 , postoperative: $3.88 ; P<.001$ ), hand to back (preoperative: 2.11 , postoperative: $3.67 ; P<.001$ ), and hand to mouth (preoperative: 2.11 ; postoperative: 3.88 ; $P<.001)$. The total Mallet score increased from 10.67 preoperatively to 19.33 postoperatively at the latest follow-up, which carried a high statistical significance $(P<.001)$. Group 1 had an average preoperative active abduction of $8.33^{\circ}$ (range, $5^{\circ}-10^{\circ}$ ), which improved to $151.1^{\circ}$ (range, $90^{\circ}-165^{\circ}$ ) postoperatively $(P<.001)$. The mean preoperative active external rotation of $0^{\circ}$ (range, $0^{\circ}-0^{\circ}$ ) was similarly improved to $24^{\circ}$ (range, $\left.20^{\circ}-25^{\circ}\right)$ postoperatively $(P<.001)$. No complications were noted during the follow-up (Fig. 2 and Fig 3.).

Group 2 (patients with only open PM tendon Z-plasty in addition to LD and TM tendon transfers) had a statistically highly significant increase in scores of abduction (preoperative: 2 , postoperative: $3.46 ; P<.001$ ), external rotation (preoperative: 2.33 , postoperative: $3.46 ; P<.001$ ), hand to head (preoperative: 2.13 , postoperative: $3.46 ; P<.001$ ), hand 


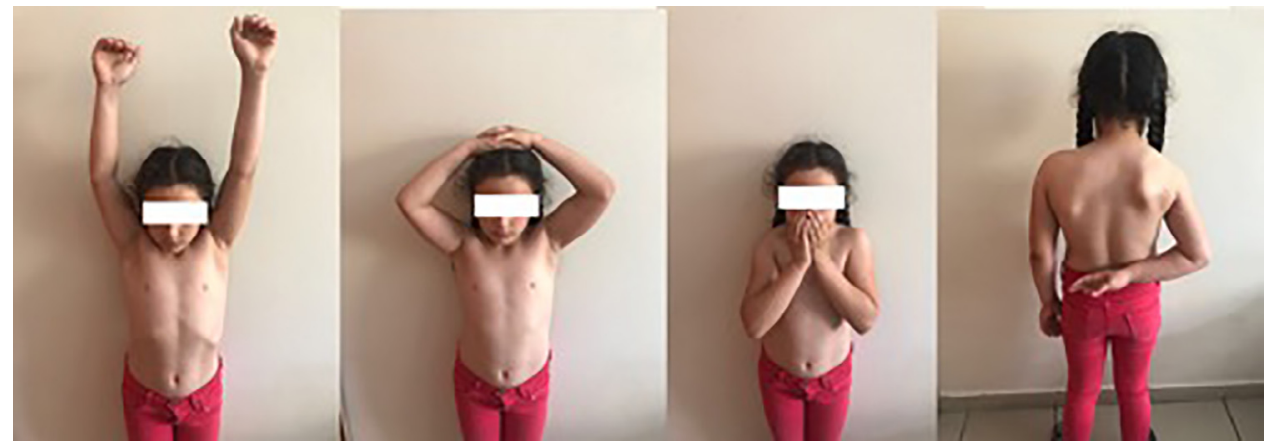

Figure 2 A patient with right-sided obstetrical brachial plexus paralysis who underwent arthroscopic subscapularis release is shown during the follow-up appointment at the third year postoperatively.

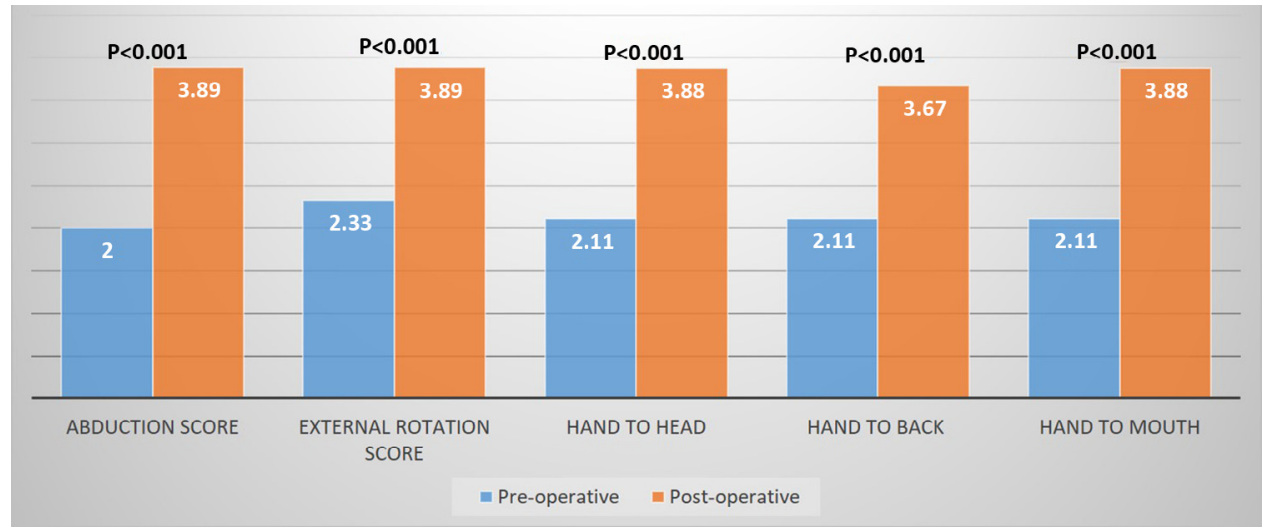

Figure 3 Preoperative and postoperative components of Mallet scores of the arthroscopic subscapularis release group.

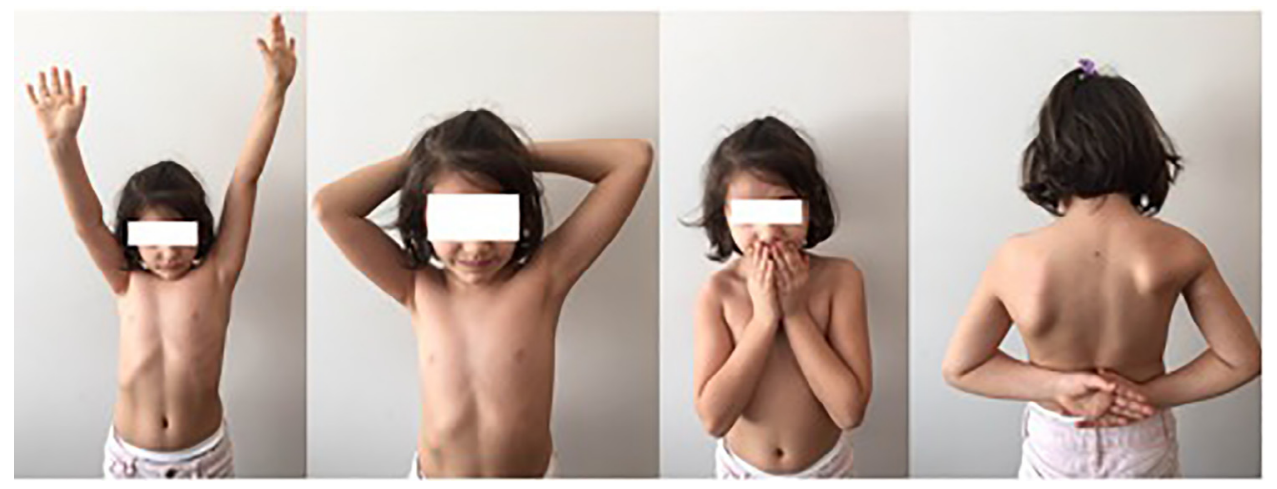

Figure 4 A patient with right-sided obstetrical brachial plexus paralysis who underwent open pectoralis major tendon Z-plasty is shown during the follow-up appointment at the third year postoperatively.

to back (preoperative: 2.2 , postoperative: $3.26 ; P<.001$ ), and hand to mouth (preoperative: 2.17 , postoperative: 3.13 ; $P<.001)$. The total Mallet score increased from 10.73 preoperatively to 16.8 postoperatively at the latest follow-up, which also carried a high statistical significance $(P<.001)$. Group 2 had an average preoperative active abduction of $9.6^{\circ}$ (range, $5^{\circ}-15^{\circ}$ ) and an average postoperative active abduction of $110.1^{\circ}$ (range, $80^{\circ}-160^{\circ}$ ), which was detected to show a remarkable improvement $(P<.001)$. A mean preoperative active external rotation of $2^{\circ}$ (range, $0^{\circ}-10^{\circ}$ ) was similarly improved to $21^{\circ}$ (range, $\left.15^{\circ}-25^{\circ}\right)$ postoperatively $(P<.001)$.
Similar to group 1, no complications were noted during the follow-up of group 2 (Fig. 4 and Fig. 5).

As the components of the Mallet scores were compared with each other between the 2 groups, the abduction $(P=.043)$, external rotation $(P=.043)$, hand to head $(P=.043)$ and hand to mouth scores $(P<.001)$ were statistically significantly better in patients who only underwent arthroscopic SS release in addition to LD and TM tendon transfers (group 1). The hand to back score was also better in group 1 than in group 2 (3.67 vs. 3.26) but was not statistically significant $(P=.060$; Fig. 6). 


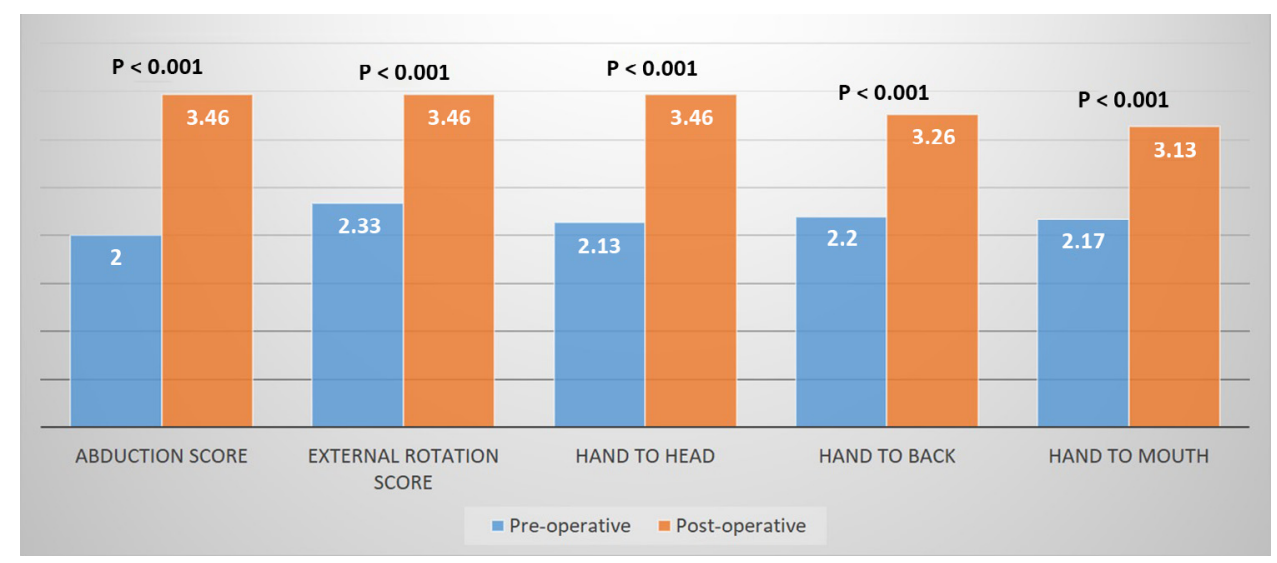

Figure 5 Preoperative and postoperative components of Mallet scores of the open pectoralis major tendon Z-plasty group.

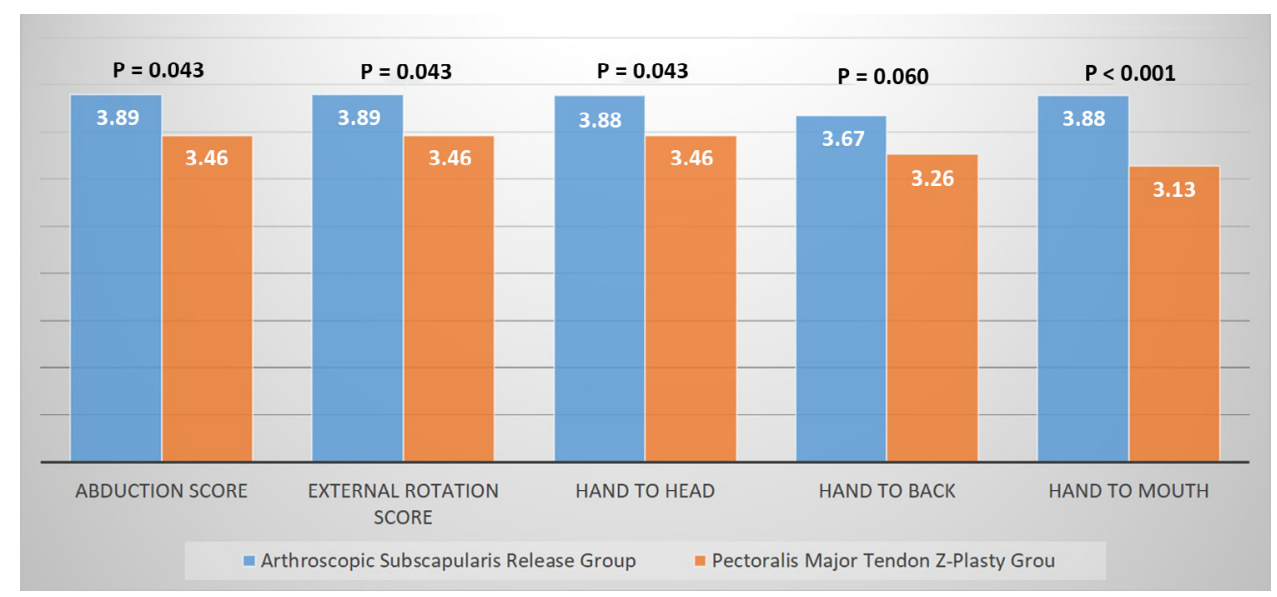

Figure 6 Postoperative comparison of components of the Mallet scores of both groups.

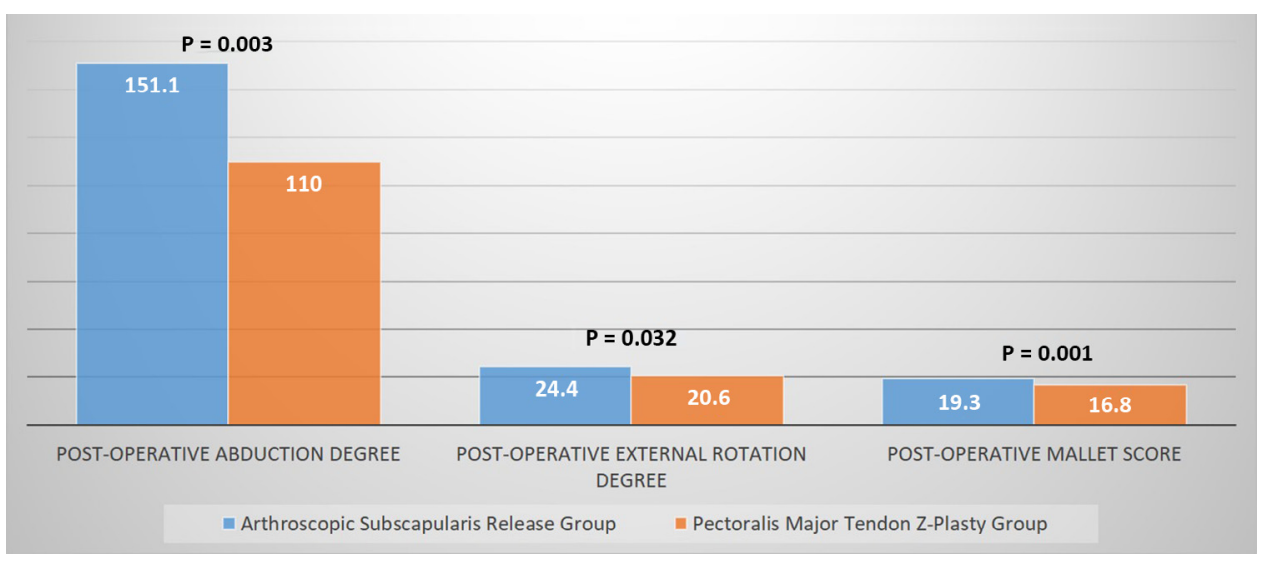

Figure 7 Postoperative comparison of abduction-external rotation degrees and total Mallet scores of both groups.

The average Mallet score of group 1 (19.3) at the latest follow-up visit was superior to that of group 2 (16.8), with a high statistical significance $(P=.001)$. In addition, the mean postoperative abduction degree of group $1\left(151.1^{\circ}\right)$ was superior to that of group $2\left(110^{\circ}\right)$, with a high statistical significance $(P=.003)$. Similarly, the mean postoperative external rotation degree of group $1\left(24^{\circ}\right)$ was also noted to be superior to that of group $2\left(21^{\circ}\right)$, with statistical significance $(P=.032$, Fig. 7$)$.

\section{Discussion}

The most important problems in patients with OBPP sequela were reported to be internal rotation contractures because of 
muscular imbalance. ${ }^{15,27,29}$ Various options of treatments have been suggested, including the transfer of LD and TM tendons, open or arthroscopic, full or partial release of SS muscle, and

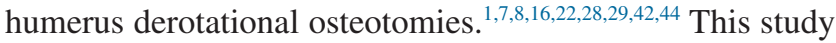
conducted a functional and radiographic comparison between internal rotation release methods. Treatment goals of OBPP sequela are keeping the shoulder reduced, eliminating the internal rotation contracture, and providing active external rotation. However, the search to find the optimal surgical treatment option for patients with OBPP is still ongoing. This is why we performed and compared 2 types of single-stage operations: LD + TM tendon transfer with arthroscopic SS release vs. LD + TM tendon transfer with open PM tendon Z-plasty.

Various combinations of different surgical procedures have been described. SS release together with the transfer of TM and LD tendons to the posterior aspect of the humerus was first described by L'Episcopo in 1934; later, Hoffer et $\mathrm{al}^{20}$ modified this technique and proposed that the LD and TM tendons should be transferred to weakened rotator cuff muscles (supraspinatus/infraspinatus) without the release of SS or PM tendons.

A significant challenge about the timing of the surgery for patients with OBPP exists in the literature. We performed arthroscopic and open procedures on patients aged 7 or younger and without any advanced glenohumeral deformity to provide the chance for growth-driven joint remodeling. In the literature, it was suggested that these children should undergo surgery as early as possible to avoid worsening of glenohumeral deformation leading to severe internal rotation contractures, which resulted in poor surgical outcomes. ${ }^{28,38,45}$ Better outcomes were reported to be expected in younger children. ${ }^{10}$

To the best of our knowledge, no studies to date have compared the effectiveness of internal rotation contraction release procedures. Most of the studies have been case series or descriptions of combined surgical techniques without any comparison. ${ }^{34,38,40}$

El-Gammal et $\mathrm{al}^{15}$ performed open SS tendon release, with or without open PM tendon lengthening, in 109 patients, Sever et al, ${ }^{33}$ Terzis et al, ${ }^{38}$ and Aydin et al ${ }^{3,4}$ performed open SS and PM tendon release together. Cohen et $\mathrm{al}^{10}$ performed tendon transfers combined with SS release in 24 patients and suggested that SS release was associated with clinical and functional improvement. Ozben et $\mathrm{al}^{27}$ performed LD plus TM tendon transfers with only open PM tendon Z-plasty, without SS release, and reported improved global shoulder function. Chen et $\mathrm{al}^{8}$ just performed tendon transfers without internal rotation contracture releases but proposed trapezius transfer if the preoperative abduction angle was below $90^{\circ}$.

It should not be forgotten that the former literature especially underlined the decrease in active internal rotation after the SS release procedures without the addition of tendon transfers underlining the insufficiency of tendon releases alone. ${ }^{16,18,33}$
Pearl et a ${ }^{29}$ performed arthroscopic SS and anterior capsular ligament release after TM and LD transfer for the surgical management of OBPP sequela and reported no recurrence of internal rotation contracture in his series of 41 patients with a follow-up of 1 to 4 years. Abid et $\mathrm{al}^{1}$ performed the SSpreserving arthroscopic release of the capsule procedure described by Kany et al, ${ }^{23}$ while releasing the superior glenohumeral and coracohumeral ligaments without releasing the SS muscle, and similarly reported no recurrence of internal rotation contracture in 14 patients with a 3-year followup duration. Our findings were also consistent with these data.

We preferred arthroscopic release of SS muscle with the aim of restoring external rotation and allowing the glenohumeral joint to remodel, to obtain better visualization of the intra-articular deformities, to easily release the anterior capsular ligaments together with the SS, and to provide a better cosmesis for the patient. Open surgery may carry a risk to pave the way for extensive scar tissue.

In this study, the modified Hoffer technique for the transfer of the LD and TM tendons was performed. They were transferred to weakened rotator cuff muscles to create the "force couple" effect so that the deltoid could work more efficiently.

During the follow-up visits, a great increase in global shoulder function was observed, without any functional and clinical deterioration and without any progressive bony deformity on the glenohumeral joint.

Most importantly, the present study concluded that compared with open PM tendon Z-plasty, arthroscopic SS release had superior outcomes in postoperative abduction and external rotation degrees, total Mallet scores, and components of the Mallet score, including abduction, external rotation, hand to head, and hand to mouth scores. In addition to cosmesis and less soft tissue dissection, arthroscopic release was noted to be superior by means of clinical and functional outcomes.

The limitations of this study were the limited number of patients, having only midterm clinical and radiographic outcomes, and having no long-term data with regard to the evaluation and comparison of the development of bony deformities.

\section{Conclusion}

Transfer of the LD together with TM tendons combined with one of the internal rotation contracture release procedures yielded good clinical and functional results in patients aged younger than 7 years, regardless of the type of release method. However, arthroscopic SS release, although requiring an experienced surgeon, resulted in better clinical and functional outcomes compared with open PM tendon Z-plasty and was therefore considered to be a less invasive and superior method, while carrying a risk of limitation of active internal rotation. 


\section{Disclaimer}

The authors, their immediate families, and any research foundations with which they are affiliated have not received any financial payments or other benefits from any commercial entity related to the subject of this article.

\section{References}

1. Abid A, Kany J, Accadbled F, Darodes P, Knorr G, Sales de Gauzy J, et al. [Arthroscopic anterior capsular release in medial contracture of the shoulder secondary to brachial plexus birth palsy. Preliminary results]. Rev Chir Orthop Reparatrice Appar Mot 2008;94:643-8 [in French]. http://dx.doi.org/10.1016/j.rco.2008.01.003

2. Al-Qattan MM. Classification of secondary shoulder deformities in obstetric brachial plexus palsy. J Hand Surg [Br] 2003;28:483-6. https://doi.org/10.1016/S0266-7681(02)00399-6

3. Aydın A, Biçer A, Özkan T, Mersa B, Özkan S, Yıldırım ZH. Does primary brachial plexus surgery alter palliative tendon transfer surgery outcomes in children with obstetric paralysis? BMC Musculoskelet Disord 2011;12:74. http://dx.doi.org/10.1186/1471-2474-12-74

4. Aydin A, Ozkan T, Onel D. Does preoperative abduction value affect functional outcome of combined muscle transfer and release procedures in obstetrical palsy patients with shoulder involvement? BMC Musculoskelet Disord 2004;5:25. http://dx.doi.org/10.1186/1471-2474 $-5-25$

5. Bahm J, Ocampo-Pavez C, Noaman H. Microsurgical technique in obstetric brachial plexus repair: a personal experience in 200 cases over 10 years. J Brachial Plex Peripher Nerve Inj 2007;2:1. http://dx.doi.org/ 10.1186/1749-7221-2-1

6. Bennett JB, Allan CH. Tendon transfers about the shoulder and elbow in obstetrical brachial plexus palsy. Instr Course Lect 2000;49:319-32.

7. Carlioz H, Brahimi L. Place of internal disinsertion of the subscapularis muscle in the treatment of obstetric paralysis of the upper limb in children]. Ann Chir Infant 1971;12:159-67 [in French].

8. Chen L, Gu YD, Hu SN. Applying transfer of trapezius and/or latissimus dorsi with teres major for reconstruction of abduction and external rotation of the shoulder in obstetrical brachial plexus palsy. J Reconstr Microsurg 2002;18:275-80. http://dx.doi.org/10.1055/s-2002-30183

9. Chuang DC, Ma HS, Wei FC. A new strategy of muscle transposition for treatment of shoulder deformity caused by obstetric brachial plexus palsy. Plast Reconstr Surg 1998;101:686-94.

10. Cohen G, Rampal V, Aubart-Cohen F, Seringe R, Wicart P. Brachial plexus birth palsy shoulder deformity treatment using subscapularis release combined to tendons transfer. Orthop Traumatol Surg Res 2010;96:334-9. http://dx.doi.org/10.1016/j.otsr.2010.02.004

11. DiTaranto P, Campagna L, Price AE, Grossman JA. Outcome following nonoperative treatment of brachial plexus birth injuries. J Child Neurol 2004;19:87-90. http://dx.doi.org/10.1177/08830738040190020101

12. Dunkerton MC. Posterior dislocation of the shoulder associated with obstetric brachial plexus palsy. J Bone Joint Surg Br 1989;71:764-6.

13. Egloff DV, Raffoul W, Bonnard C, Stalder J. Palliative surgical procedures to restore shoulder function in obstetric brachial palsy. Critical analysis of Narakas' series. Hand Clin 1995;11:597-606.

14. El-Gammal TA, El-Sayed A, Kotb MM, Ragheb YF, Saleh WR, Elnakeeb RM, et al. Total obstetric brachial plexus palsy: results and strategy of microsurgical reconstruction. Microsurgery 2010;30:169-78. http://dx.doi.org/10.1002/micr.20726

15. El-Gammal TA, Saleh WR, El-Sayed A, Kotb MM, Imam HM, Fathi NA. Tendon transfer around the shoulder in obstetric brachial plexus paralysis: clinical and computed tomographic study. J Pediatr Orthop 2006;26:641-6. http://dx.doi.org/10.1097/01.bpo.0000229975.86188.c4
16. Fairbank H. Birth palsy: subluxation of the shoulder-joint in infants and young children. Lancet 1913;1:6

17. Gilbert A. Long-term evaluation of brachial plexus surgery in obstetrical palsy. Hand Clin 1995;11:583-94, discussion 594-85.

18. Gilbert A, Brockman R, Carlioz H. Surgical treatment of brachial plexus birth palsy. Clin Orthop Relat Res 1991;(264):39-47.

19. Hoeksma AF, Ter Steeg AM, Dijkstra P, Nelissen RG, Beelen A, de Jong BA. Shoulder contracture and osseous deformity in obstetrical brachial plexus injuries. J Bone Joint Surg Am 2003;85-A:316-22.

20. Hoffer MM, Wickenden R, Roper B. Brachial plexus birth palsies. Results of tendon transfers to the rotator cuff. J Bone Joint Surg Am 1978;60:691-5.

21. Javid M, Shahcheraghi GH. Shoulder reconstruction in obstetric brachial plexus palsy in older children via a one-stage release and tendon transfers. J Shoulder Elbow Surg 2009;18:107-13. http://dx.doi.org/10.1016/ j.jse.2008.06.013

22. Kambhampati SB, Birch R, Cobiella C, Chen L. Posterior subluxation and dislocation of the shoulder in obstetric brachial plexus palsy. J Bone Joint Surg $\operatorname{Br}$ 2006;88:213-9. http://dx.doi.org/10.1302/0301 $-620 x .88 b 2.17185$

23. Kany J, Kumar HA, Amaravathi RS, Abid A, Accabled F, de Gauzy JS, et al. A subscapularis-preserving arthroscopic release of capsule in the treatment of internal rotation contracture of shoulder in Erb's palsy (SPARC procedure). J Pediatr Orthop B 2012;21:469-73. http:// dx.doi.org/10.1097/BPB.0b013e328353a19f

24. Michelow BJ, Clarke HM, Curtis CG, Zuker RM, Seifu Y, Andrews DF. The natural history of obstetrical brachial plexus palsy. Plast Reconstr Surg 1994;93:675-80, discussion 681.

25. Moukoko D, Ezaki M, Wilkes D, Carter P. Posterior shoulder dislocation in infants with neonatal brachial plexus palsy. J Bone Joint Surg Am 2004;86-A:787-93.

26. Nath RK, Paizi M. Improvement in abduction of the shoulder after reconstructive soft-tissue procedures in obstetric brachial plexus palsy. J Bone Joint Surg Br 2007;89:620-6. http://dx.doi.org/10.1302/0301 $-620 x .89 b 5.18403$

27. Ozben H, Atalar AC, Bilsel K, Demirhan M. Transfer of latissimus dorsi and teres major tendons without subscapularis release for the treatment of obstetrical brachial plexus palsy sequela. J Shoulder Elbow Surg 2011;20:1265-74. http://dx.doi.org/10.1016/j.jse.2011.01.004

28. Pagnotta A, Haerle M, Gilbert A. Long-term results on abduction and external rotation of the shoulder after latissimus dorsi transfer for sequelae of obstetric palsy. Clin Orthop Relat Res 2004;(426):199-205.

29. Pearl ML. Arthroscopic release of shoulder contracture secondary to birth palsy: an early report on findings and surgical technique. Arthroscopy 2003;19:577-82.

30. Pedowitz DI, Gibson B, Williams GR, Kozin SH. Arthroscopic treatment of posterior glenohumeral joint subluxation resulting from brachial plexus birth palsy. J Shoulder Elbow Surg 2007;16:6-13. http://dx.doi.org/ 10.1016/j.jse.2006.04.008

31. Pichon F, Carlioz H. Disinsertion of the subscapularis muscle in the treatment of obstetric paralysis of the upper limb (author's transl)]. Chir Pediatr 1979;20:135-41 [in French].

32. Price AE, Grossman JA. A management approach for secondary shoulder and forearm deformities following obstetrical brachial plexus injury. Hand Clin 1995;11:607-17.

33. Sever JW. Obstetric paralysis-its cause and treatment. Can Med Assoc J 1920;10:141-61.

34. Shenaq SM, Kim JY, Armenta AH, Nath RK, Cheng E, Jedrysiak A. The surgical treatment of obstetric brachial plexus palsy. Plast Reconstr Surg 2004;113:54e-67e.

35. Smith NC, Rowan P, Benson LJ, Ezaki M, Carter PR. Neonatal brachial plexus palsy. Outcome of absent biceps function at three months of age. J Bone Joint Surg Am 2004;86-A:2163-70.

36. Soucacos PN, Vekris MD, Zoubos AB, Johnson EO. Secondary reanimation procedures in late obstetrical brachial plexus palsy patients. Microsurgery 2006;26:343-51. http://dx.doi.org/10.1002/ micr.20249 
37. Strombeck C, Krumlinde-Sundholm L, Forssberg H. Functional outcome at 5 years in children with obstetrical brachial plexus palsy with and without microsurgical reconstruction. Dev Med Child Neurol 2000;42:148-57.

38. Terzis JK, Kokkalis ZT. Outcomes of secondary shoulder reconstruction in obstetrical brachial plexus palsy. Plast Reconstr Surg 2008;122:181222. http://dx.doi.org/10.1097/PRS.0b013e31818cc1fc

39. Terzis JK, Kokkalis ZT. Primary and secondary shoulder reconstruction in obstetric brachial plexus palsy. Injury 2008;39(Suppl. 3):S5-14. http://dx.doi.org/10.1016/j.injury.2008.06.001

40. van Kooten EO, Fortuin S, Winters HA, Ritt MJ, van der Sluijs HA. Results of latissimus dorsi transfer in obstetrical brachial plexus injury. Tech Hand Upper Extrem Surg 2008;12:195-9. http://dx.doi.org/ 10.1097/BTH.0b013e318176b32f

41. van Ouwerkerk WJ, van der Sluijs JA, Nollet F, Barkhof F, Slooff AC. Management of obstetric brachial plexus lesions: state of the art and future developments. Child's Nerv Syst 2000;16:63844.

42. Waters PM. Comparison of the natural history, the outcome of microsurgical repair, and the outcome of operative reconstruction in brachial plexus birth palsy. J Bone Joint Surg Am 1999;81:64959.

43. Waters PM. Obstetric brachial plexus injuries: evaluation and management. J Am Acad Orthop Surg 1997;5:205-14.

44. Waters PM, Bae DS. The effect of derotational humeral osteotomy on global shoulder function in brachial plexus birth palsy. J Bone Joint Surg Am 2006;88:1035-42. http://dx.doi.org/10.2106/jbjs.e.00680

45. Waters PM, Peljovich AE. Shoulder reconstruction in patients with chronic brachial plexus birth palsy. A case control study. Clin Orthop Relat Res 1999;(426):144-52.

46. Zancolli EA. Classification and management of the shoulder in birth palsy. Orthop Clin North Am 1981;12:433-57. 